\title{
Fluorescence characteristics of human Barrett tissue specimens grafted on chick chorioallantoic membrane
}

\author{
Jasmin A. Holz ${ }^{1}$ - David F. Boerwinkel ${ }^{2}$ - Sybren L. Meijer ${ }^{3}$ - Mike Visser ${ }^{3}$. \\ Ton G. van Leeuwen ${ }^{1}$ • Jacques J. G. H. M. Bergman ${ }^{2} \cdot$ Maurice C. G. Aalders $^{1}$
}

Received: 10 May 2015 / Accepted: 18 November 2015 / Published online: 4 December 2015

(C) The Author(s) 2015. This article is published with open access at Springerlink.com

\begin{abstract}
To improve (pre)malignant lesion identification in Barrett's esophagus (BE), recent research focuses on new developments in fluorescence imaging and spectroscopy to enhance tissue contrast. Our aim was to validate the chorioallantoic membrane (CAM) model as a preclinical tool to study the fluorescence characteristics such as autofluorescence and exogenously induced fluorescence of human Barrett's tissue. Therefore, esophageal biopsy specimens from Barrett's patients were freshly grafted onto the CAM of fertilized hen's eggs to simulate the in vivo situation. The BE biopsy specimens stayed between 1 and 9 days on the CAM to study the persistence of vitality. Fluorescence spectroscopy was performed using six excitation wavelengths $(369,395,400$, 405, 410, $416 \mathrm{~nm}$ ). Obtained autofluorescence spectra were compared with in vivo spectra of an earlier study. Exogenous administration of 5-aminolevulinic-acid to the biopsy specimens was followed by fluorescence spectroscopy at several time points. Afterwards, the biopsy specimens were harvested and histologically evaluated. In total, 128 biopsy specimens obtained from 34 patients were grafted on the CAM. Biopsy specimens which stayed on average 1.7 days on the CAM were still vital. Autofluorescence spectra of the specimens correlated well with in vivo spectra. Administered 5-
\end{abstract}

Jasmin A. Holz

j.a.holz@amc.uva.nl

1 Department of Biomedical Engineering and Physics, Academic Medical Center, Meibergdreef 9,

1105AZ Amsterdam, The Netherlands

2 Department of Gastroenterology and Hepatology, Academic Medical Center, Meibergdreef 9, 1105AZ Amsterdam, The Netherlands

3 Department of Pathology, Academic Medical Center, Meibergdreef 9, 1105AZ Amsterdam, The Netherlands aminolevulinic-acid to the biopsy specimens showed conversion into protoporphyrin-IX. In conclusion, we showed that grafting freshly collected human BE biopsy specimens on the CAM is feasible. Our results suggest that the CAM model might be used to study the fluorescence behavior of human tissue specimens. Therefore, the CAM model might be a preclinical research tool for new photosensitizers.

Keywords Barrett's esophagus · Chorioallantoic membrane . Early detection of cancer · Fluorescence spectroscopy ·

5-aminolevulinic-acid $\cdot$ Protoporphyrin-IX

\section{Introduction}

Patients with Barrett's esophagus (BE) are recommended to undergo regular surveillance endoscopy to detect malignant lesions at an early stage. Esophageal adenocarcinoma develops from premalignant stages of dysplasia, which can be histologically graded. These precursor lesions are amenable to curative and minimally invasive endoscopy therapy, due to the low risk of lymph node metastasis. With a low morbidity and mortality compared to esophagectomy and an excellent 5-year survival rate, the timely detection of early dysplasia is of great clinical importance [1-4].

Early changes in tissue during the progression into malignancy occur at a (sub)cellular level and consist of morphological and chemical changes which cannot be seen during standard endoscopic diagnostic procedures such as white light endoscopy. Recent developments to improve real-time diagnostics focus on imaging such as high-resolution white light endoscopy (HR-WLE), confocal endomicroscopy, narrowband imaging (NBI), and autofluorescence imaging (AFI); the latest suffers from a high false-positive rate [5-11]. Although a lot of effort has been put in these new 
technologies, none are currently able to clearly discriminate in real time healthy from premalignant tissue during standard surveillance endoscopy. But these (sub)cellular changes in premalignant tissue do affect the optical absorption and scattering properties of the tissue which might be detected by fluorescence spectroscopy [12-15]. In addition, photosensitizers, such as 5-aminolaevulinic acid (5-ALA) may be administered to enhance the tissue contrast $[16,17]$. Administration of 5-ALA leads to the formation of the fluorescent protoporphyrin-IX (PpIX). Metabolic differences between tissue types cause concentration variations. At specific times, the concentration of PpIX inside the (pre)malignant tissue [13] is higher compared to normal tissue levels, which shows as increased fluorescence and is therefore often used to improve the discrimination between dysplastic and non-dysplastic epithelium. This so-called photodiagnosis provides real-time information that can be used to red flag areas of interest during medical procedures for the detection of (early) cancer.

For developing new photosensitizers and the characterization of current sensitizers, usually cell experiments and/or animal experiments are used. The physiology and overall drugcell interactions are far from the in vivo situation. Animal experiments are expensive and cumbersome. A model which is very suitable for studying the localization of the particles and the treatment efficacy is the chick embryo chorioallantoic membrane (CAM) model which uses the well-vascularized chorioallantoic membrane of fertilized chicken eggs on which tumors can be implanted [18-20]. After implantation, angiogenesis allows the tumor to grow. Because of the easy accessibility of the systemic and tumor circulation, it is possible to illuminate the CAM and study the fluorescence yield and, if applicable, treatment effect. The CAM model is the bridge between the preliminary cell experiments and the in vivo tumor-bearing animal models. To mimic the in vivo situation even better, we grafted freshly excised tissue biopsies directly onto the CAM, thereby maintaining the heterogeneity and tumor architecture, which are lost in homogenous cultures of tumor tissue.

Our aim was to validate the CAM model as a preclinical tool to study the fluorescence characteristics of human Barrett's tissue and therefore the potential use to test future photosensitizers. First, we studied the vitality of freshly human BE tissue biopsies grafted onto the CAM by examining the hematoxylin and eosin (H\&E)-stained slices of the harvested biopsies. Furthermore, the autofluorescence spectra obtained from the grafted tissue were compared with in vivo autofluorescence spectra, and the well-known 5-ALA was topically administered to test the CAM model for the usage of photosensitizer. As light source, a multi-wavelength spectroscopy system consisting of several discrete excitation wavelengths around the 405-nm Soret absorption peak of PpIX was used. This setting was chosen to find the optimal excitation wavelength of PpIX in Barrett's tissue to optimize new imaging and spectroscopy technologies for improved premalignant lesion identification.

\section{Materials and methods}

\section{Spectroscopy system}

A custom-made spectroscopy system was developed (2M Engineering Ltd., Veldhoven, the Netherlands) for endoscopic in vivo measurements comprising a LED with $369 \mathrm{~nm}$ (FWHM of $16 \mathrm{~nm}$ ) and laser diodes at 395, 400, 405, 410, and $416 \mathrm{~nm}$ [15]. The system was connected to an optical fiber probe which delivered the excitation light to the tissue and the fluorescence light back to the connected spectrometer USB4000 (Ocean Optics Inc., Dunedin, Florida, USA) and laptop for spectral recording. The distal fiber probe tip had an outer diameter of $2 \mathrm{~mm}$ and an inner functional diameter of $0.82 \mathrm{~mm}$ which consisted of a bundle of 30 fibers, thus 15 illumination and 15 collection fibers. Proximal, the 15 collection fibers were aligned to the $200-\mu \mathrm{m}$ entrance slit of the spectrometer. The biopsy specimens were placed perpendicular under the tip of the probe, thus mimicking the in vivo endoscopic setting.

\section{CAM model}

Fertilized hen's eggs obtained from Drost Loosdrecht BV (Loosdrecht, The Netherlands) were incubated for 3 days in a Polyhatch incubator (Brinsea Products Inc., Titusville, Florida, USA) at $38-39{ }^{\circ} \mathrm{C}, 60-80 \%$ humidity, rotating every $1 \mathrm{~h}$. At embryonic day 3, a volume of $2-3 \mathrm{~mL}$ albumen was removed from the egg using a $21-G$ needle injected into the air pocket of the egg in order to lower the level of the CAM inside the egg. A window of approximately $1.5 \mathrm{~cm}^{2}$ was cut into the outer shell on top of the egg in order to gain access to the CAM, using blades and scissors. After checking if the embryo was alive, the window was covered with transparent plastic and the egg was placed in a hatcher (Brinsea Products Inc., Titusville, Florida, USA) at $37.5{ }^{\circ} \mathrm{C}, 50-70 \%$ humidity. When outside the hatcher, the eggs were handled on a heating plate at $37^{\circ} \mathrm{C}$ in a laminar flow hood.

\section{Patient selection and biopsy grafting onto the CAM}

Patients scheduled for surveillance endoscopy of non-dysplastic BE (NDBE) or work-up or treatment of early Barrett's neoplasia at the department of Gastroenterology and Hepatology of the Academic Medical Center (AMC) Amsterdam were included. The Medical Ethics Committee of the AMC Amsterdam approved the study and all included patients were informed and signed a consent form. Biopsies of lesions suspicious for dysplasia within the BE and of endoscopically unsuspicious areas of BE were obtained from 34 patients (two to four biopsies per 
patient). Immediately after the biopsies were obtained, they were transferred into preheated $37.5^{\circ} \mathrm{C}$ transport medium (DMEM, FCS, PenStrep, L.Glut, Fungizone).

At embryonic day 6 of incubation, the egg was placed in the laminar flow hood on a heating plate at $37^{\circ} \mathrm{C}$. The plastic cover was lifted from the egg, and the CAM was cleaned at the location for tissue grafting with ethanol-treated lens paper. The freshly obtained biopsy specimens were subsequently spread on a gloved finger and with the help of two pairs of tweezers carefully spread and placed on the CAM. One biopsy specimen was grafted per egg. The transparent cover was closed, and the egg put back in the hatcher.

\section{Spectroscopy procedure}

The egg was placed in the laminar flow hood and positioned under the spectroscopy set-up. All measurements were performed in a dark room. The optical fiber probe was placed about $1 \mathrm{~mm}$ above the biopsy specimen, followed by sequential illumination by all light sources and recording of the fluorescence spectra, including a dark measurement (all light sources off). Subsequently, spectra were recorded adjacent to the biopsy specimen and on the CAM only which was approximately $2 \mathrm{~cm}$ from the biopsy specimen. Each saved spectrum was composed of the average of three measurements per site. For the fluorescence spectroscopy measurements with 5-ALA administration, $20 \mu \mathrm{L}$ of $10 \mathrm{mM}$ 5-ALA (Sigma Chemical Co.) dissolved in $0.9 \% \mathrm{NaCl}$ solution was topically administered to each biopsy specimen. Fluorescence spectroscopy was performed at several time points between $0 \mathrm{~h}$ before and $23 \mathrm{~h}$ after 5-ALA administration. After the last measurement, the biopsy specimens were removed from the CAM and fixed in formalin, embedded in paraffin, and cut and stained with hematoxylin and eosin (H\&E). Histopathological assessment of the biopsy specimens was performed by an expert GI pathologist. Biopsies which were considered vital were classified into two groups, suspicious for dysplasia called "dysplastic" and not suspicious for dysplasia called "non-dysplastic." Finally, the embryo was terminated by high-dose isoflurane.

\section{Data analysis}

The obtained autofluorescence spectra of the biopsy specimens on the CAM were analyzed in the same way as the in vivo spectra which we obtained in an earlier study [15]. Pearson's correlation coefficient $r$ and the coefficient of determination $r^{2}$ were calculated to assess the relationship between the autofluorescence spectra from the biopsy specimens and the in vivo spectra.

From all emission spectra, obtained from the biopsy specimens used for 5-ALA measurements, the corresponding dark spectrum was subtracted. The intensity ratio $I_{636} / I_{600}$ of the PpIX fluorescence peak at $636 \mathrm{~nm}$ to a reference emission wavelength at $600 \mathrm{~nm}$ was calculated for each spectrum.
This corrects for variations in applied laser power, probe positioning, and system noise. The mean intensity ratios, the standard deviation (SD), and the standard error of the mean (SEM) were calculated. The statistical relevance of differences in intensity ratios per excitation wavelength and time point was determined by repeated measures one-way ANOVA with subsequent Bonferroni correction using Prism 5 (GraphPad Software Inc., La Jolla, California, USA). The results of Bonferroni's multiple comparison test were considered significant when the $p$ value was $<0.05$.

\section{Imaging}

Images were obtained from the biopsy specimens on the CAM using a Dino-Lite digital microscope in cross-polarized white light imaging mode (AM413ZT, AnMo Electronics Corp., Hsinchu, Taiwan) before and after 5-ALA administration. Fluorescence imaging was performed to obtain an overview of the PpIX distribution on the biopsy specimens and CAM. Fluorescence images were obtained at blue light illumination ranging from 400 to $430 \mathrm{~nm}$ (Crime-lite ${ }^{\circledR} 2$, Foster+Freeman Ltd., Evesham, UK). A digital camera (Nikon D40X) with a long pass filter (GG455, Foster+Freeman) in front of the lens was used to allow only the detection of emitted fluorescence at longer wavelengths than $435 \mathrm{~nm}$.
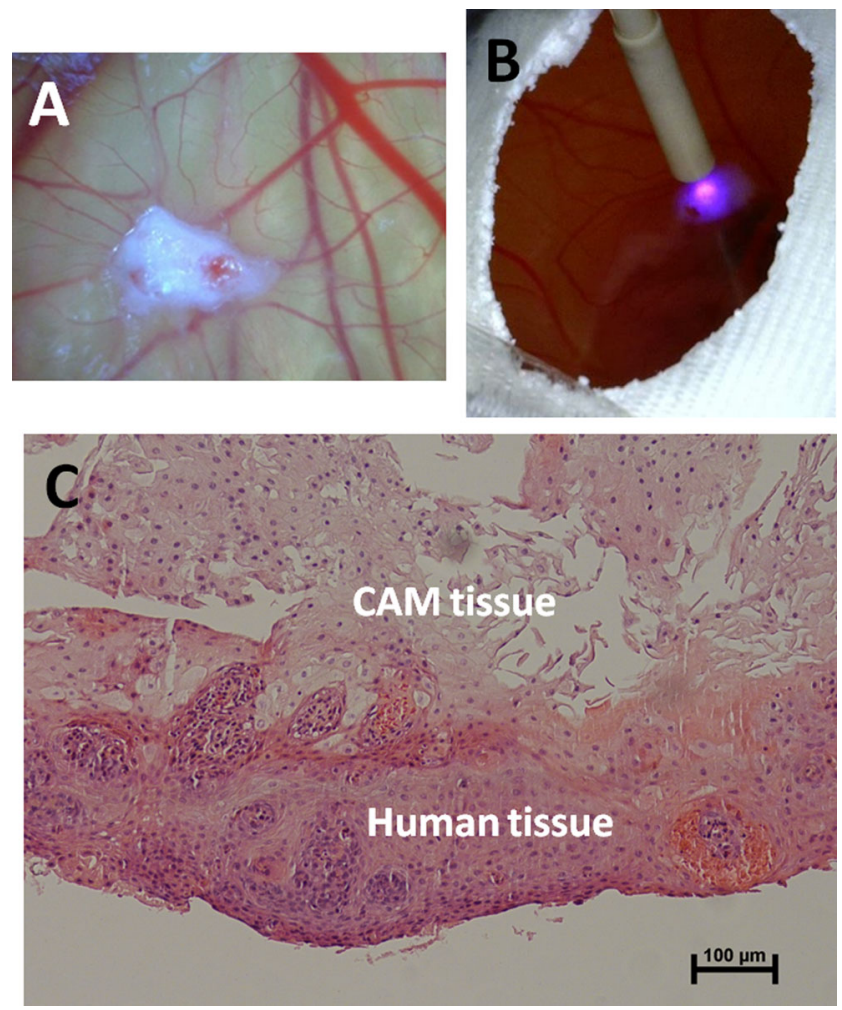

Fig. 1 a Image of Barrett's esophageal biopsy specimen on the CAM; b fluorescence spectroscopy performed on a biopsy specimen; $\mathbf{c}$ white light microscope image with $\times 10$ magnification of $\mathrm{H} \& \mathrm{E}$-stained slice of a human biopsy specimen which stayed 1 day on the CAM 
Table 1 Coefficient of determination $r^{2}$ of averaged ex vivo versus in vivo autofluorescence spectra for non-dysplastic and dysplastic esophageal tissue

\begin{tabular}{lll}
\hline & $\begin{array}{l}\text { Non-dysplastic } \\
\text { ex vivo vs. in vivo }\end{array}$ & $\begin{array}{l}\text { Dysplastic ex } \\
\text { vivo vs. in vivo }\end{array}$ \\
\hline $369 \mathrm{~nm}$ & 0.977 & 0.976 \\
$395 \mathrm{~nm}$ & 0.961 & 0.987 \\
$405 \mathrm{~nm}$ & 0.961 & 0.991 \\
$410 \mathrm{~nm}$ & 0.969 & 0.991 \\
$416 \mathrm{~nm}$ & 0.968 & 0.991 \\
\hline
\end{tabular}

\section{Results}

\section{Biopsy assessment and autofluorescence spectra evaluation}

In total, 63 biopsy specimens, obtained from 25 Barrett's patients, stayed between 1 and 9 days on the CAM. Twenty-six biopsy specimens showed necrosis and were not classified histopathologically. Fourteen other histology slices did not contain enough tissue for an assessment. From the remaining 23 biopsy specimens, 9 were classified as not suspicious for dysplasia (non-dysplastic) thus squamous or non-dysplastic Barrett's esophagus (NDBE) and 14 as suspicious for dysplasia (dysplastic). The 23 biopsies which could be classified stayed on average 1.7 days on the CAM. Figure 1a shows an image of a Barrett's biopsy specimen on the CAM.

Autofluorescence spectroscopy was performed, see Fig. 1b, on 8 of the 9 non-dysplastic biopsies and on 10 of the 14 dysplastic-classified biopsies. Pearson's correlation coefficient $r$ and the coefficient of determination $r^{2}$ (Table 1) were calculated to correlate ex vivo and in vivo averaged autofluorescence spectra obtained with the same system
[15]. The ex vivo dysplastic autofluorescence spectra correlated well with the in vivo (HGIN/CA) spectra with an $r^{2}$ value of around 0.99 . The non-dysplastic spectra correlated less with the in vivo (IM) spectra having an $r^{2}$ value of around 0.97 . Figure 2 shows the averaged autofluorescence spectra at 395 $\mathrm{nm}$ excitation from in vivo (HGIN/CA) and ex vivo (dysplastic) esophageal tissue with an $r^{2}$ value of 0.987 .

Figure 1c shows the H\&E-stained slice of a human biopsy specimen which stayed 1 day on the CAM. In the upper part, the CAM cells are seen, and in the lower part, the human tissue seemed to be vital and attached to the CAM tissue.

\section{5-ALA-induced fluorescence spectra evaluation}

A total of 65 biopsies, obtained from 20 Barrett's patients, stayed between 2 and 4 days on the CAM and were then examined with 5-ALA-induced spectroscopy. Histopathological evaluation of the harvested biopsies was not possible for 25 biopsy specimens due to intensive necrosis and 10 more slices contained not enough tissue for an assessment. From the remaining 30 biopsy specimens, 12 were classified as not suspicious for dysplasia (non-dysplastic) thus squamous or NDBE and 18 as suspicious for dysplasia (dysplastic). Three spectra of non-dysplastic biopsy specimens were excluded from the analysis due to measurement issues. Table 2 gives an overview of the spectra included for the analysis at all six excitation wavelengths obtained from 12 patients.

Figure 3 shows typical emission spectra with subtracted dark spectrum before normalization at all six excitation wavelengths, $6 \mathrm{~h}$ after 5-ALA administration, of a biopsy specimen (dysplastic) on the CAM (a), the CAM adjacent to the biopsy specimen (b), and the CAM only (d). Fluorescence spectra of the biopsy specimens on the CAM showed PpIX fluorescence peaks at $636 \mathrm{~nm}$. Maximum autofluorescence was observed
Fig. 2 Averaged

autofluorescence spectra at 395-

$\mathrm{nm}$ excitation from in vivo

(HGIN/CA) and ex vivo

(dysplastic) esophageal tissue

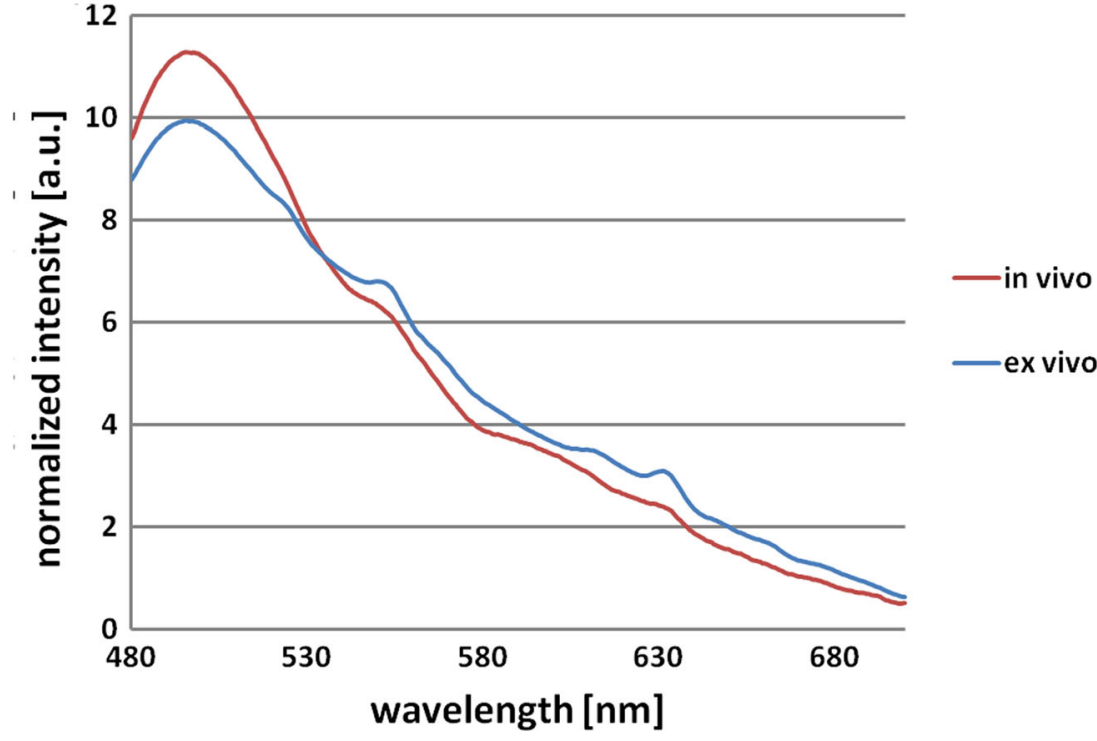


Table 2 Number of analyzed human biopsy specimens on the CAM per time point and their histological classification

\begin{tabular}{llll}
\hline Time point & $\begin{array}{l}\text { Not suspicious } \\
\text { for dysplasia }\end{array}$ & $\begin{array}{l}\text { Suspicious } \\
\text { for dysplasia }\end{array}$ & Sum \\
\hline $0 \mathrm{~h}$ & 9 & 18 & 27 \\
$1.5 \mathrm{~h}$ & 9 & 18 & 27 \\
$4.5 \mathrm{~h}$ & 8 & 16 & 24 \\
$6 \mathrm{~h}$ & 6 & 11 & 17 \\
$23 \mathrm{~h}$ & 2 & 7 & 9 \\
\hline
\end{tabular}

around $500 \mathrm{~nm}$. In contrast, the emission spectra adjacent to the biopsy specimens showed negligible autofluorescence and a clear PpIX fluorescence profile. The main difference between the biopsy specimens and the adjacent fluorescence was that the CAM did not show porphyrin fluorescence at the 620- and 680-nm emissions. Fluorescence spectra of the CAM only revealed slightly less autofluorescence compared to the biopsy specimen and negligible PpIX fluorescence compared to the biopsy specimens and the surrounding CAM.

The PpIX intensity ratios $I_{636} / I_{600}$ did not differ significantly between dysplastic and non-dysplastic tissue. Therefore, and due to the low amount of biopsy specimens classified as non-dysplastic, we decided to analyze all biopsy specimens (Table 2) per time point and excitation wavelength in one group, to which we refer further as $\mathrm{BE}$ tissue.

The intensity ratios $I_{636} / I_{600}$ of BE tissue showed increased PpIX fluorescence with increasing excitation wavelength and time point (Fig. 4a). Bonferroni's multiple comparison tests of all pairs of time points showed a significant increase in the intensity ratios at 0 vs. 6 h, 0 vs. 23 h, 1.5 vs. 23 h, and 4.5 vs. $23 \mathrm{~h}$ at all excitation wavelengths. Intensity ratios at $369-\mathrm{nm}$ excitation were significantly lower compared to $405-, 410-$, and 416-nm excitation at all time points. The intensity ratios at $410-$ and 416-nm excitation at $4.5 \mathrm{~h}$ were significantly higher compared to $0 \mathrm{~h}$. At $4.5 \mathrm{~h}$ after 5-ALA administration, the PpIX intensity ratios at 416-nm excitation were significantly higher compared to all other excitation wavelengths except $410 \mathrm{~nm}$. Each measurement at $4.5 \mathrm{~h}$ after 5-ALA administration separately showed that in $96 \%(23 / 24)$ of the cases, the highest PpIX fluorescence intensity ratios were obtained at $410-\mathrm{nm}$ (63\%) and 416-nm (33\%) excitations. The lowest intensity ratios were obtained at 369-nm excitation in $96 \%(23 / 24)$ of the cases. The intensity ratios at $410-\mathrm{nm}$ excitation were 2.3 times higher (SD: \pm 0.8 ) compared to 369-nm excitation.

The intensity ratios $I_{636} / I_{600}$ on the CAM adjacent to the BE tissue (Fig. 4b) tend to increase faster and became higher compared to the BE tissue. There was a significant increase of the intensity ratios on the CAM adjacent to the $\mathrm{BE}$ tissue between time point 0 vs. 1.5 h, 0 vs. 4.5 h, 0 vs. 6 h, and a significant decrease at time points 4.5 vs. $23 \mathrm{~h}$ and 6 vs. $23 \mathrm{~h}$ at all excitation wavelengths except $369 \mathrm{~nm}$. At $23 \mathrm{~h}$ after 5-ALA administration, the PpIX fluorescence was back at baseline level $(t=0)$. The PpIX fluorescence on the CAM only (Fig. 4c) increased significantly after $6 \mathrm{~h}$, but those intensity ratios were negligible (five to seven times lower) compared to the intensity ratios obtained from the $\mathrm{BE}$ tissue or adjacent to it.

Fluorescence imaging was performed to obtain an overview of the PpIX distribution on the BE tissue and CAM. Fluorescence images (Fig. 5) taken several hours after 5-ALA administration showed first a strong red fluorescence surrounding the $\mathrm{BE}$ tissue and with increasing time increasing fluorescence of the BE tissue and decreasing fluorescence of the surrounding CAM.

\section{Discussion}

The evaluation of the CAM model showed that biopsy specimens were still vital after 1.7 days on the CAM, on average. The lower intensities of the ex vivo autofluorescence spectra around $500 \mathrm{~nm}$ might be caused by the different measurement procedures; in vivo, the probe was in contact with the tissue site under investigation, whereas ex-vivo, the probe did not touch the biopsy specimen. Whereas, the higher intensities in the rest of the ex vivo autofluorescence spectra might be caused by lower blood absorption compared to the in vivo situation. Despite these deviations, the overall correlation of the ex vivo with the in vivo autofluorescence spectra showed good resemblance.
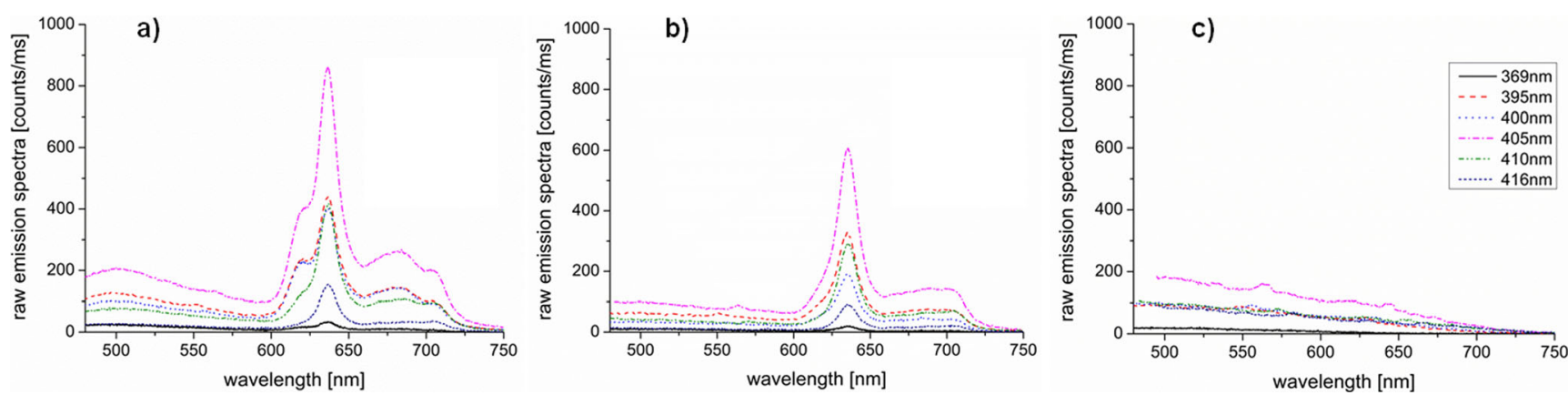

Fig. 3 Typical emission spectra with subtracted dark spectrum at all six excitation wavelengths, $6 \mathrm{~h}$ after 5-ALA administration, of a biopsy specimen (dysplastic) on the CAM (a), the CAM adjacent to the biopsy specimen (b), and the CAM only (c) 

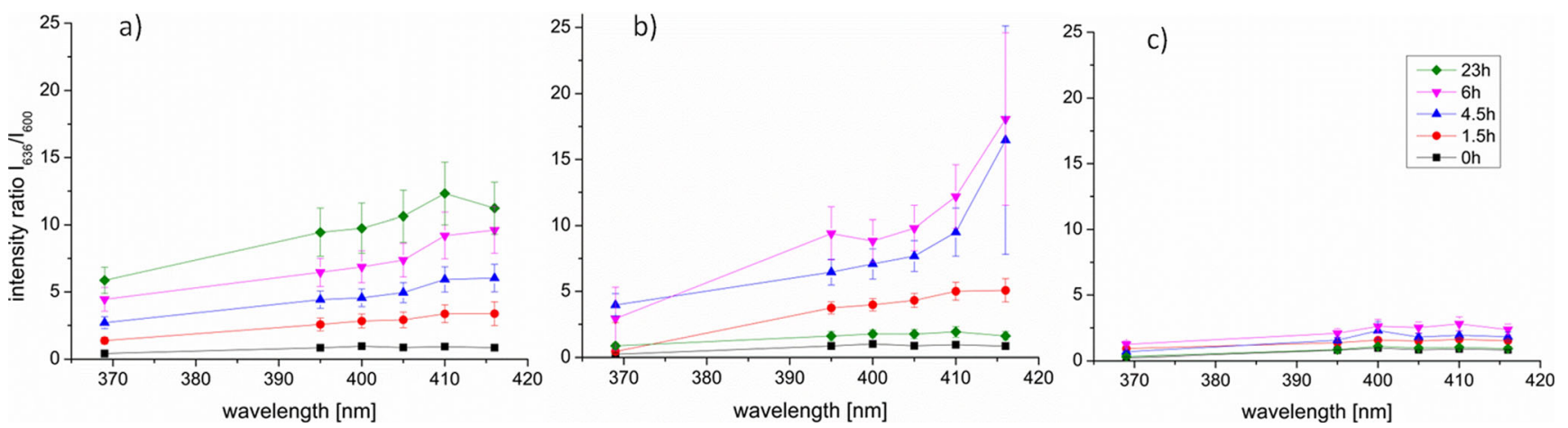

Fig. 4 Mean PpIX intensity ratios $I_{636} / I_{600}$ with standard error of the mean at $0,1.5,4.5,6$, and $23 \mathrm{~h}$ after 5-ALA administration obtained from the BE tissue on the CAM (a), the CAM adjacent to the BE tissue (b), and the CAM only (c)

Previous research using the CAM model in combination with 5-ALA focused on tumor specimens and cell lines [21-24]. The observed conversion into PpIX in our study suggests the existence of a functional metabolism within the BE tissue. As expected, the lowest PpIX intensities were induced with the shortest wavelength, $369 \mathrm{~nm}$, which is about $30 \mathrm{~nm}$ below the Soret band of PpIX. The highest PpIX intensities were induced at 410- and 416-nm excitations without a significant difference between them.

In our study, BE tissue showed increased PpIX fluorescence with increasing time, which is in agreement with known in vivo observations. The $\mathrm{BE}$ tissue showed no significantly reduced PpIX fluorescence after $23 \mathrm{~h}$, which indicates a slower PpIX clearance compared to in vivo observations [25-28]. This increased clearance time might be caused by the topical bolus administration of ALA. The excessive ALA is not removed, and therefore, the tissue exposure is expected to be longer compared to in vivo conditions. Furthermore, the ALA diffusion into the tissue and redistribution within the egg might be prolonged in the CAM model. The in vivo kinetics were already studied [25-28]; therefore, we focused on studying the CAM model as a preclinical approach for fluorescence diagnostics with special interest in photosensitizer. This preclinical model benefits from no patient hazard, and it is a more physiological approach then studying cells in vitro. The fluorescence of the CAM adjacent to the $\mathrm{BE}$ tissue showed a faster increase and decrease of
$3 h$

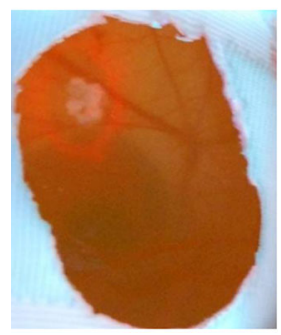

$6 h$

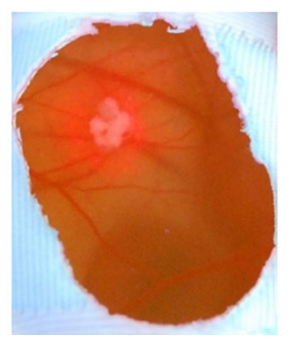

$23 \mathrm{~h}$

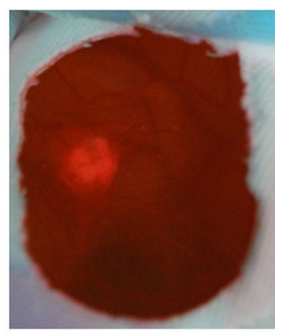

Fig. 5 Fluorescence images of BE tissue on the CAM at 3, 6, and $23 \mathrm{~h}$ after 5-ALA administration induced PpIX, whereas the PpIX fluorescence and autofluorescence of the CAM only showed negligible intensities. Fluorescence images showed that the high PpIX fluorescence adjacent to the biopsy specimens was due to the topical application of 5-ALA surrounding the biopsy specimens.

Although the CAM model was applicable for our research purpose, the spectroscopy measurements had several limitations. The spectroscopy set-up used a mounted probe, to ensure stable measurements. However, due to movements of the embryo and therefore repositioning of the $\mathrm{BE}$ tissue under the probe, equal distances and angles of the measurements cannot be assured and sometimes measurements needed to be repeated. The histological classification showed that around $40 \%$ of the grafted biopsies did not stay vital on the CAM and therefore needed to be excluded from the analysis. This necessitates the usage of an increased amount of biopsies or an optimized protocol, for example, by leaving the biopsy specimens a maximum of 2 days on the CAM or daily administration of growth factors and/or medium to the tissue on the CAM which may prolong tissue vitality.

Differentiation between BE tissue and the CAM was possible, due to the differences in spectral shape, 5-ALA kinetics, and the wavelength-dependent intensity ratios. The main difference between $\mathrm{BE}$ tissue and the CAM spectra was that the CAM did not show porphyrin fluorescence at the 620- and 680-nm emissions (Fig. 3). This fluorescence might be caused by the formation of water-soluble porphyrins [29].

In conclusion, we showed that grafting freshly collected human BE biopsy specimens on the CAM is feasible. Our results suggest that the CAM model might be used to study fluorescence diagnostics such as autofluorescence and induced fluorescence behavior of human biopsy specimens on the CAM as a preclinical research tool. Further preclinical research is recommended, with an increased amount of biopsies from non-dysplastic and dysplastic BE tissue, to assess the potential in tissue discrimination. Furthermore, with the CAM model keeping human tissue vital for a few days, the responses to new photosensitizer might be evaluated. Besides that, our current fluorescence spectroscopy system can be modified by adding a white light source for additional 
reflectance spectroscopy which had shown its potential in endoscopic detection of dysplasia [30-32].

Acknowledgments This research was funded by the 7th Framework Programme for Research and Technological Development of the European Union; grant agreement number 231993 (EDOCAL).

Compliance with ethical standards All procedures performed in studies involving human participants were in accordance with the ethical standards of the institutional and/or national research committee and with the 1964 Helsinki declaration and its later amendments or comparable ethical standards. Informed consent was obtained from all individual participants included in the study. The authors declare that they have no conflict of interest.

Open Access This article is distributed under the terms of the Creative Commons Attribution 4.0 International License (http:// creativecommons.org/licenses/by/4.0/), which permits unrestricted use, distribution, and reproduction in any medium, provided you give appropriate credit to the original author(s) and the source, provide a link to the Creative Commons license, and indicate if changes were made.

\section{References}

1. Ell C, May A, Gossner L, Pech O, Gunter E, Mayer G, Henrich R, Vieth M, Muller H, Seitz G, Stolte M (2000) Endoscopic mucosal resection of early cancer and high-grade dysplasia in Barrett's esophagus. Gastroenterology 118:670-677

2. Ell C, May A, Pech O, Gossner L, Guenter E, Behrens A, Nachbar L, Huijsmans J, Vieth M, Stolte M (2007) Curative endoscopic resection of early esophageal adenocarcinomas (Barrett's cancer). Gastrointest Endosc 65:3-10

3. Behrens A, Pech O, Graupe F, May A, Lorenz D, Ell C (2011) Barrett's adenocarcinoma of the esophagus: better outcomes through new methods of diagnosis and treatment. Dtsch Arztebl Int 108:313-319

4. Westerterp M, Koppert LB, Buskens CJ, Tilanus HW, ten Kate FJ, Bergman JJ, Siersema PD, van Dekken H, van Lanschot JJ (2005) Outcome of surgical treatment for early adenocarcinoma of the esophagus or gastro-esophageal junction. Virchows Arch 446:497-504

5. Borovicka J, Fischer J, Neuweiler J, Netzer P, Gschossmann J, Ehmann T et al (2006) Autofluorescence endoscopy in surveillance of Barrett's esophagus: a multicenter randomized trial on diagnostic efficacy. Endoscopy 38:867-872

6. DaCosta RS, Wilson BC, Marcon NE (2007) Fluorescence and spectral imaging. Sci World J 7:2046-2071

7. Curvers WL, Singh R, Song LM, Wolfsen HC, Ragunath K, Wang K et al (2008) Endoscopic tri-modal imaging for detection of early neoplasia in Barrett's oesophagus: a multi-centre feasibility study using high-resolution endoscopy, autofluorescence imaging and narrow band imaging incorporated in one endoscopy system. Gut 57:167-172

8. Curvers WL, Herrero LA, Wallace MB, Wong Kee Song LM, Ragunath K, Wolfsen HC et al (2010) Endoscopic tri-modal imaging is more effective than standard endoscopy in identifying early-stage neoplasia in Barrett's esophagus. Gastroenterology 139:1106-1114

9. Curvers WL, van Vilsteren FG, Baak LC, Bohmer C, Mallant-Hent RC, Naber AH et al (2011) Endoscopic trimodal imaging versus standard video endoscopy for detection of early Barrett's neoplasia: a multicenter, randomized, crossover study in general practice. Gastrointest Endosc 73:195-203

10. Boerwinkel DF, Holz JA, Aalders MC, Visser M, Meijer SL, van Berge Henegouwen MI, Weusten BL, Bergman JJ (2013) Third- generation autofluorescence endoscopy for the detection of early neoplasia in Barrett's esophagus: a pilot study. Dis Esophagus 27(3):276-84

11. Boerwinkel DF, Holz JA, Kara MA, Meijer SL, Wallace MB, Wong Kee Song LM, Ragunath K, Wolfsen HC, Iyer PG, Wang KK, Weusten BL, Aalders MC, Curvers WL, Bergman JJ (2014) Effects of autofluorescence imaging on detection and treatment of early neoplasia in patients with Barrett's esophagus. Clin Gastroenterol Hepatol 12:774-781

12. Bigio IJ, Mourant JR (1997) Ultraviolet and visible spectroscopies for tissue diagnostics: fluorescence spectroscopy and elasticscattering spectroscopy. Phys Med Biol 42:803-814

13. Wagnieres GA, Star WM, Wilson BC (1998) In vivo fluorescence spectroscopy and imaging for oncological applications. Photochem Photobiol 68:603-632

14. Ramanujam N (2000) Fluorescence spectroscopy of neoplastic and non-neoplastic tissues. Neoplasia 2:89-117

15. Holz JA, Boerwinkel DF, Meijer SL, Visser M, Van Leeuwen TG, Aalders MC, Bergman JJ (2013) Optimized endoscopic autofluorescence spectroscopy for the identification of premalignant lesions in Barrett's oesophagus. Eur J Gastroenterol Hepatol 25(12):14421449

16. Fotinos N, Campo MA, Popowycz F, Gurny R, Lange N (2006) 5aminolevulinic acid derivatives in photomedicine: characteristics, application and perspectives. Photochem Photobiol 82:994-1015

17. Krammer B, Plaetzer K (2008) ALA and its clinical impact, from bench to bedside. Photochem Photobiol Sci 7:283-289

18. Ausprunk DH, Knighton DR, Folkman J (1975) Vascularization of normal and neoplastic tissue grafted to the chick choriollantois. Role of host and preexisting graft vessels. Am J Pathol 79:597-618

19. Deryugina EI, Quigley JP (2008) Chick embryo chorioallantoic membrane model systems to study and visualize human tumor cell metastasis. Histochem Cell Biol 130(6):1119-1130

20. Xiao X, Zhou X, Ming H, Zhang J, Huang G, Zhang Z, Li P (2015) Chick chorioallantoic membrane assay: a $3 \mathrm{D}$ animal model for study of human nasopharyngeal carcinoma. PLoS One 10(6): e0130935

21. Hoppenheit C, Huttenberger D, Foth HJ, Spitzer WJ, Reichert TE, Muller-Richter UD (2006) Pharmacokinetics of the photosensitizers aminolevulinic acid and aminolevulinic acid hexylester in oro-facial tumors embedded in the chorioallantois membrane of a hen's egg. Cancer Biother Radiopharm 21:569-578

22. Hornung R, Hammer-Wilson MJ, Kimel S, Liaw LH, Tadir Y, Berns MW (1999) Systemic application of photosensitizers in the chick chorioallantoic membrane (CAM) model: photodynamic response of CAM vessels and 5-aminolevulinic acid uptake kinetics by transplantable tumors. J Photochem Photobiol B 49:41-49

23. Piffaretti F, Novello AM, Kumar RS, Forte E, Paulou C, NowakSliwinska P, van den Bergh H, Wagnieres G (2012) Real-time, in vivo measurement of tissular $\mathrm{pO} 2$ through the delayed fluorescence of endogenous protoporphyrin IX during photodynamic therapy. $\mathrm{J}$ Biomed Opt 17:115007

24. Zenzen V, Zankl H (2003) Protoporphyrin IX-accumulation in human tumor cells following topical ALA- and h-ALA-application in vivo. Cancer Lett 202:35-42

25. Hinnen P, de Rooij FW, Hop WC, Edixhoven A, van Dekken H, Wilson JH, Siersema PD (2002) Timing of 5-aminolaevulinic acidinduced photodynamic therapy for the treatment of patients with Barrett's oesophagus. J Photochem Photobiol B 68:8-14

26. Loh CS, MacRobert AJ, Bedwell J, Regula J, Krasner N, Bown SG (1993) Oral versus intravenous administration of 5-aminolaevulinic acid for photodynamic therapy. Br J Cancer 68:41-51

27. Loh CS, Vernon D, MacRobert AJ, Bedwell J, Bown SG, Brown SB (1993) Endogenous porphyrin distribution induced by 5aminolaevulinic acid in the tissue layers of the gastrointestinal tract. J Photochem Photobiol B 20:47-54 
28. Bedwell J, MacRobert AJ, Phillips D, Bown SG (1992) Fluorescence distribution and photodynamic effect of ALA-induced PP IX in the DMH rat colonic tumour model. Br J Cancer 65:818-824

29. Dietel W, Pottier R, Pfister W, Schleier P, Zinner K (2007) 5-aminolaevulinic acid (ALA) induced formation of different fluorescent porphyrins: a study of the biosynthesis of porphyrins by bacteria of the human digestive tract. J Photochem Photobiol B 86:77-86

30. Wallace MB, Wax A, Roberts DN, Graf RN (2009) Reflectance spectroscopy. Gastrointest Endosc Clin N Am 19(2):233-242
31. Zhu Y, Fearn T, Mackenzie G, Clark B, Dunn JM, Bigio IJ, Bown SG, Lovat LB (2009) Elastic scattering spectroscopy for detection of cancer risk in Barrett's esophagus: experimental and clinical validation of error removal by orthogonal subtraction for increasing accuracy. J Biomed Opt 14(4):044022

32. Wallace MB, Perelman LT, Backman V, Crawford JM, Fitzmaurice M, Seiler M, Badizadegan K, Shields SJ, Itzkan I, Dasari RR, Van Dam L, Feld MS (2000) Endoscopic detection of dysplasia in patients with Barrett's esophagus using light-scattering spectroscopy. Gastroenterology 119(3):677-682 